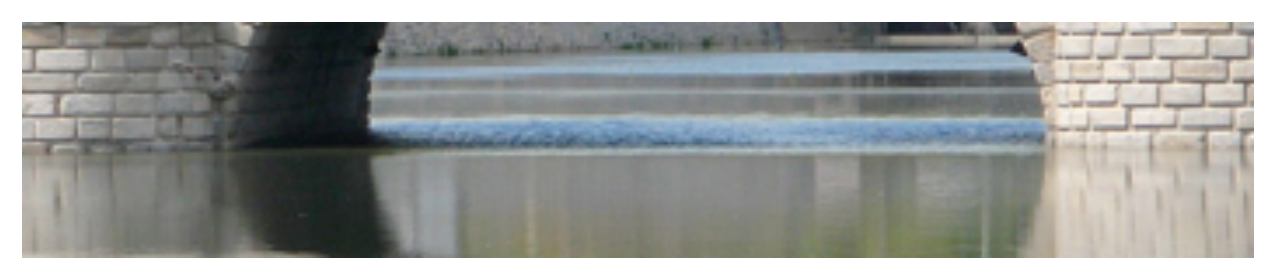

\title{
Conversaciones digitales: las mediaciones del uso de la Internet en los telecentros de los proyectos Faróis do Saber y Paranavegar ${ }^{1}$
}

\section{Digital Conversations: the Mediations of Internet Use in the Telecenters of the Projects Faróis do Saber and Paranavegar}

\author{
Juciano de Sousa Lacerda ${ }^{(*)}$ \\ Universidade Federal do Rio Grande do Norte - Brasil \\ juciano@cchla.ufrn.br
}

\begin{abstract}
Resumen
La investigación trata del proceso de mediatización digital en los telecentros de acceso público y gratuito, teniendo la tecnicidad como mediación constitutiva de los significados producidos por usuarios de Internet en la vida cotidiana de las condiciones materiales y simbólicas que configuran el ambiente. El recorte empírico sitúa las experiencias de la inclusión digital de la ciudad de Curitiba (Faróis do Saber) y el gobierno de Paraná (Paranavegar). Metodológicamente, se trabaja con Webgrafía, Mediografía $y$ entrevistas en profundidad, que hizo posible una asignación del ciberespacio navegado y
\end{abstract}

\begin{abstract}
This research explores the digital mediatisation process in free and public access telecenters, with technicality as constituent mediation of significations produced by Internet users in the everyday life of material and symbolic conditions, which configures the ambience. The empirical approach focuses on the digital inclusion experiences of the city of Curitiba (Faróis do Saber, Portuguese for knowledge lighthouse) and Paraná State Government (Paranavegar). The methodological set includes Webgraphy, Mediagraphy and deep interviews,
\end{abstract}

\footnotetext{
1 Esta es una versión revisada y ampliada del documento presentado en el Grupo de Investigación "Comunicación para la Ciudadanía" de la X Reunión de Grupos de Investigación en Comunicación, el evento componente XXXIII Congreso Brasileño de Ciencias de la Comunicación, 2010.
} 
el tiempo que se queda en cada sitio. Fueron 5650 minutos de navegación de 136 usuarios de Internet, la mayoría niños, adolescentes y jóvenes, que caracterizaron el ambiente de los telecentros como un lugar donde la espacialidad digital es principalmente de conversación.

Palabras-clave: mediaciones comunicacionales, tecnicidad, telecentros comunitarios, redes sociotécnicas, políticas de inclusión digital. which allowed to measure the browsed cyberspace and the time spent in each site. In 5650 minutes of navigation by 136 Internet users, mostly children, teenagers and youth, who featured the ambience in telecenters as a place where the digital spatiality is mainly for conversation purposes.

Keywords: communicational mediation, technicality, community telecenters, sociotechnical networks, digital inclusion policies. 


\section{LA TECNICIDAD COMO MEDIACIÓN}

El teórico Jesús Martín-Barbero ha estado llevando a cabo una revisión del concepto de mediación para establecer la tecnicidad como una dimensión constitutiva del proceso de producción de sentido (MartínBarbero, 2001b: 9-13). Inspirado por Marcel Mauss, también pone la técnica en el mismo lugar fonético de la racionalidad o la sociabilidad.

"Pues cada cultura, por pequeño que sea el número de sus miembros, tiene un sistema técnico que se basa en una determinada "tendencia técnica", que es lo que nombra la palabra tecnicidad, dando así el salto a pensar el carácter estructurador que la tecnología tiene en la sociedad" (Martín-Barbero, 2004: 25).

La visión de tecnicidad de Martín-Barbero se basa también en Heidegger, para quien "la técnica no es pues un mero medio, la técnica es un modo de salir de lo oculto" (Heidegger, 2001: 14). Preguntar por la técnica, para Heidegger, es la posibilidad de desvelamiento. Ver la técnica como "interpelación liberadora" y no como algo que se desarrolla de modo ciego o contra el cual tenemos que rebelarnos (Heidegger, 2001: 24). Al mismo tiempo, la técnica es un "territorio de misterio", ya que no sólo revela, sino que también puede ocultar u olvidar. "Todo hacer salir lo oculto pertenece a un albergar y a un ocultar. Pero ocultado está, y siempre está ocultándose, lo que libera, el misterio" (Heidegger, 2001: 23). Heidegger llama a la visión instrumental de la técnica como "algo caducado" puesto que la tecnicidad no es ni "un mero hacer del hombre ni tan solo un simple medio dentro de los límites de este hacer” (Heidegger, 2001: 20).

Tal como señala Martín-Barbero (2004), inspirado por Heidegger, la técnica no es sólo fabricación, sino producción que revela la dimensión humana de la innovación, del hacer existir lo nuevo, al mismo tiempo que amenaza hacernos olvidar. ¿Y qué corremos el riesgo de olvidar? "De que la verdad de la existencia humana no se agota en el saber ligado a la técnica pues hay otro saber que es el saber-del-ser" (Martín-Barbero, 2004: 27).

Por lo tanto, pensar la tecnicidad mediática en términos de finalidad es permanecer en el punto de vista instrumental, cuando deberíamos buscar el desvelamiento, es decir, el sentido de las técnicas mediáticas que nos lleva a pensar en formas de apropiación. Siempre teniendo en cuenta que "el medio no se limita a vehicular o traducir las representaciones existen-

Mediaciones Sociales, № 8, I semestre 2011, pp. 127-154. ISSN electrónico: 1989-0494. DOI: $10.5209 / \mathrm{rev} \_M E S O .2011 . n 8.6$ 
tes, ni puede tampoco sustituirlas, sino que ha entrado a constituir una escena fundamental de la vida pública” (Martín-Barbero, 2004: 31 ).

Cuando se trata específicamente de la política como una de las mediaciones (instituciones) sociales, Martín-Barbero señala una mediación que la técnica realiza entre la política y la vida cotidiana de los ciudadanos. Desde el punto de vista de Heidegger, Martín-Barbero sugiere que la técnica mediática pone de manifiesto una profunda desconexión entre los partidos políticos ante las nuevas sensibilidades. Los partidos políticos quedarían hoy desplazados socialmente, porque habían perdido el control "de la configuración simbólica de la representación” (Martín-Barbero, 2004: 34).

Sin embargo, la constitución de la técnica mediática (tecnicidad) no representa el fin de la política (mirada que podemos ampliar para otros tipos de mediación institucional), pero

"la reconfiguración de las mediaciones que constituyen sus modos de interpelación de los sujetos y de representación de los vínculos que cohesionan la sociedad. Mediaciones que se están tornando cada día socialmente más productivas (en el sentido en el que Heidegger entiende la producción) pero cuya producción permanece impensada” (Martín-Barbero, 2004: 36).

También existe el riesgo de sobrevaloración de la "mediación tecnológica” (Orozco, 2006) en relación con otras mediaciones sociales en el campo de la comunicación. Este es el caso de Guillermo Orozco que asevera el debilitamiento de las mediaciones propias de las instituciones sociales como la escuela o el Estado, que eran importantes para el público en su producción de sentido. Y que en el desordenamiento de las mediaciones tradicionales "la mediación tecnológica logra importancia quizá excesiva, mientras que otras mediaciones casi desaparecen, o hacen trinchera en el fundamentalismo” (Orozco, 2006: 88-89).

Es decir, él no se da cuenta -como Martín-Barbero (2004)- de una reconfiguración de las mediaciones, en lugar de su desaparición o reclusión, dada la configuración de un "ecosistema comunicativo" que no se limita a las máquinas y los medios, pero sí amplia a

"nuevos lenguajes, sensibilidades, saberes y escrituras, por la hegemonía de la experiencia audiovisual sobre la tipográfica, y por la reintegración de la imagen al campo de la producción de conocimiento. Todo lo cual está inci- 
diendo tanto sobre lo que entendemos por comunicar como sobre las figuras del convivir y el sentido de lazo social” (Martín-Barbero, 2004: 36).

Es decir, estos nuevos lenguajes, sensibilidades, conocimientos y escrituras (Piscitelli, 2005; Martín-Barbero, 2004) son producidas en la compleja articulación entre la oferta mediática y la recepción. Son nuevas posibilidades de gramáticas de producción y reconocimiento desarrolladas en la intersección de los formatos mediáticos industriales (con su lógica del mercado) con las matrices culturales (Martín-Barbero, 2001a). Cuando alguien -en el eje de la producción- desarrolla una plataforma para la interacción entre personas en el ambiente digital, piensa en términos de interfaz, o sea, de representación (Johnson, 2001: 4), y no solamente como una racionalidad matemática del código binario, sino en cómo estos códigos pueden representar un contexto reconocible por los usuarios de Internet. Es decir, los formatos digitales se desarrollan a partir de experiencias culturales, de las matrices que constituyen al sujeto que la produce, pues el mundo-objeto de la tecnología debe ser considerado como perteneciente al mundo de la cultura (Johnson, 2001). Por lo tanto, medios digitales y sus interfaces son de una determinada cultura o se desarrollaron a partir de un modelo cultural específico.

En el caso de la entrada del ordenador en un determinado contexto sociocultural -en función de políticas de lucha contra la brecha digital que recomiendan la presencia física del equipo como la posibilidad para el desarrollo-, sus efectos sobre la comunidad local no se producen como causalidad. Los significados producidos, las formas de apropiación son complejos y dependen del contexto de la mediación.

Por lo tanto, si queremos entender la tecnicidad como mediación es necesario ver que el flujo demanda una nueva visión, ya no en términos de finalidad, causa y consecuencia -la perspectiva lineal- sino en términos de la circularidad de las causas, de la recursividad. La mediación, que implica el movimiento de significados, es el movimiento de sentido más allá de una secuencia de dos etapas (Silverstone, 2002: 33). Así, si una vez la sociedad ha creado estos dispositivos tecnológicos por demandas de comunicación específicas, estos objetos, en sus usos, requieren nuevas formas y modalizaciones de interacción. Como señala Milton Santos sobre las ciudades como objetos técnicos modernos, la co-presencia e intercambios también son condicionados por la infraestructura (2002: 319). Este factor condicionante, aunque sea visto como una racionalidad estructurada y estructuran- 
te (Bourdieu, 2003), que limita y controla los márgenes de flujo de la oferta, también puede ser "roto" por el mismo proceso de circulación, por quien está en la acción en el papel de recepción y consumo. La contraparte del flujo se puede ver, por ejemplo, en lo que Milton Santos calificó como acción silenciosa, o, a veces, ruidosa, de los pobres, o aquellos para los que no fue pensada -o excluidos de- la racionalidad técnica.

"Los pobres abren un nuevo debate, inédito, a veces silencioso, a veces ruidoso, con las poblaciones y las cosas ya presentes. Es así que reevalúan la tecnosfera y psicosfera, encontrando nuevos usos y finalidades para objetos y técnicas, así como nuevas articulaciones prácticas y nuevas normas, en la vida social y afectiva" (Santos, 2002: 326).

La producción de nuevos usos y resignificación de finalidades nos obligan a ver el consumo como un lugar de lucha en que no es la posesión de objetos lo que está en juego. El consumo es también un lugar de producción de sentido, que se caracteriza por los usos que dan forma a los objetos sociales "y en los cuales se inscriben demandas y dispositivos de acción procedentes de diferentes competencias culturales” (MartínBarbero, 2001a: 302).

Y en el proceso de producción de sentidos, como afirma MartínBarbero, se recupera el proceso de reconocimiento como una interpelación, que cuestiona la constitución de los individuos y grupos, y fortalece la sociabilidad.

“Todos ellos hacen y rehacen a sí mismos en el tejido simbólico de las interpelaciones, de los reconocimientos. Todo sujeto está sujeto a otro y es a la vez sujeto para alguien. Es la dimensión viva de la sociabilidad a través y manteniendo la dimensión institucional” (Martín-Barbero, 2001a: 316).

La mediación, por tanto, no es individual, sino que implica la labor de instituciones, grupos y tecnologías (Silverstone, 2002). 


\section{NAVEGACIÓN EN INTERNET: LA CONVERSACIÓN DIGITAL}

En nuestra investigación de doctorado la propuesta era describir e interpretar el proceso de mediatización en los telecentros ${ }^{2}$ de acceso público y gratuito, teniendo la tecnicidad como mediación constitutiva de las significaciones producidas por los usuarios de Internet en la vivencia cotidiana de las condiciones simbólicas y materiales que dan forma al ambiente de los telecentros. Nuestra premisa fue investigar proyectos de inclusión digital desarrollados como política pública (Assumpção 2001; Oliva, 2003; Menou et al, 2004; Silveira, 2005, Warschauer, 2006), que nos llevó a seleccionar dos experiencias de inclusión digital, una de la Ciudad de Curitiba, llamada Faróis do Saber ${ }^{3}$ y otra del Gobierno de Paraná: el proyecto Paranavegar ${ }^{4}$.

Para coincidir con la noción de ambiente ${ }^{5}$, hemos desarrollado una metodología en circuito capaz de registrar espacialidades física, personal y

2 Nuestra comprensión de los telecentros para esta investigación se ha limitado a ambientes mediático-comunicacionales vinculados a un proyecto de inclusión digital de una institución pública, realizado por instituciones relacionadas con el desarrollo comunitario y social, y desarrollado por las organizaciones que participan en la vida de los barrios -como asociaciones de vecinos, centros de apoyo para los jóvenes, organizaciones comunitarias, asociaciones culturales y benéficas, centros de refuerzo educacional, etc.- ubicados en localidades con problemas socioeconómicos (Robinson, 2000). El espacio debe servir a los diversos grupos comunitarios, la mejora de sus intereses sociales y económicos, como la ayuda mutua y apoyo social en línea, búsqueda de trabajo, el acceso a la información libre, ir de compras y contactos comerciales a través de la red (Loader, 2004; Castells, 2003, 2004).

3 El proyecto es coordinado por el Departamento Municipal de Educación con el apoyo de Instituto de Informática de Curitiba (ICI), con 26000 personas registradas a finales de 2007. De los 45 Faróis do Saber, dos se encuentran en la región central de Curitiba, y en el Farol del Bosque de Alemán no hay telecentro. Los 43 restantes se encuentran en regiones diagnosticados por el Departamento de Educación de Curitiba como los de más necesidad, en los suburbios de la ciudad, donde las personas no tienen acceso a Internet. El tiempo de uso es de una hora por turno por persona. Los usuarios, al hacer el registro, pasan a tener una dirección de correo electrónico proporcionada por la alcaldía.

4 El proyecto es coordinado por la Secretaría Especial para Asuntos Estratégicos (SEAE) y realizado por la Compañía de Informática del Paraná (Celepar), con cerca de 44000 personas registradas en el estado a finales de 2007. Hay 110 telecentros en todo el estado, especialmente en ciudades con bajo Índice de Desarrollo Humano (IDH), beneficiando a 87000 personas por mes en 97 ciudades del estado. El telecentro se instaló el primer 14 de mayo de 2003, aniversario de la ciudad de Ventania, el $10^{\circ}$ índice de desarrollo humano más bajo de Paraná. En Curitiba, hay ocho telecentros del proyecto Paranavegar. El tiempo de uso es de 30 minutos por turno.

5 En nuestra investigación, tomamos el concepto de ambiente como un lugar que pone en relación (Bateson, 1998) distintas semiosferas (Lotman, 1996), en el que operan o se establecen

Mediaciones Sociales, № 8, I semestre 2011, pp. 127-154. ISSN electrónico: 1989-0494.

DOI: 10.5209/rev_MESO.2011.n8.6 
digital en relación (Bateson, 1998). En el análisis, identificamos la conexión entre la espacialidad personal, física y digital en un ecosistema complejo. Un ambiente en que los usuarios de la Internet -en su mayoría niños y adolescentes- tienen por objeto crear estrategias creativas de interacción en línea, desarrollando maneras astuciosas de aplicar los escasos recursos disponibles (Certeau, 2000).

Hemos desarrollado dos estrategias metodológicas, la Mediografía y la Webgrafía, y completamos con las entrevistas realizadas en profundidad. El punto de partida para el desarrollo de la Webgrafía fue la metáfora de Todd Gitlin (2005) de "torrente" o "diluvio" de los medios de comunicación. Inmerso en una enorme cantidad de información diaria, estamos desarrollando un conjunto de prácticas de calificación y de rutina de lo que tomamos como algo significativo, en efecto una especie de mapa de navegación. Tras él, casi siempre estrictamente, podemos ignorar el resto del vórtice informativo. Son nuestras "estrategias de navegación", que se desarrollan a partir de las mediaciones que nos constituyen y dejan sus huellas en la forma en que tornamos propio, re-organizamos y re-programamos el caos informativo, así que tiene sentido para nosotros. "Con el fin de controlar lo incontrolable, cultivamos las estrategias de navegación que, cuando se consolidan y se convierten en habituales, con buen criterio se pueden llamar 'estilos'” (Gitlin, 2005: 146).

Así, delante de la serie casi infinita de posibilidades para navegar por Internet, siempre desarrollamos una rutina de ambientes favoritos, a que siempre volvemos, a menudo dentro de una misma secuencia lógica. Este sería nuestro ciberespacio personal, el resultado de las negociaciones de significado en nuestras mediaciones constitutivas. En los telecentros, procuramos registrar los ambientes en que navegaban los usuarios de Internet de los telecentros Farol do Saber Aristides Vinholes, Farol do Saber Telêmaco Borba y Paranavegar Vila Real. Para eso, desarrollamos una hoja de cálculo en que se pueda registrar minuto a minuto los sitios nave-

límites, en el proceso de entendimiento mutuo y la integración en un dado mundo cultural común, que provocan tanto un acercamiento entre los diferentes espacios culturales como la producción de diferencias, especializaciones dentro de esos espacios. Por lo tanto, pensamos el ambiente de los telecentros como un lugar de relación entre distintas semiosferas: el espacio digital, espacio personal y espacio de los objetos técnicos (arquitectura). Cada uno es parte de un área de especial importancia, con su gramática y formas de organizarse, pero hay una frontera que las pone en contacto: el telecentro como ambiente mediático-comunicacional, como una semiosfera de intersección.

Mediaciones Sociales, № 8, I semestre 2011, pp. 127-154. ISSN electrónico: 1989-0494. DOI: 10.5209/rev_MESO.2011.n8.6 
gados por las páginas web, en un intervalo de una hora, límite de tiempo para cada persona en el telecentro. La estrategia hizo posible una asignación del ciberespacio navegado, de hecho, y el tiempo de permanencia en los ambientes. Fueron 5650 minutos de navegación de 136 usuarios de Internet. En la Mediografía del espacio/tiempo de los telecentros, el diario de campo fue esencial para la descripción de las acciones que realizaban los usuarios de Internet en el período navegado y documentado en la Webgrafía. Pesó a nuestro favor el hecho de que la posición de las computadoras ha permitido identificar los sitios a distancia. En cada período, hicimos el recuerdo a la gente sobre la investigación.

Las entrevistas en profundidad nos permitieron comparar las experiencias de los usuarios de Internet y la forma de dotar de significado a ellas. En total, hemos identificado un ambiente caracterizado por una atmósfera de solidaridad en las relaciones entre personas que compartían la misma espacialidad física, cuyas interacciones ampliaban la vivencia del espacio digital, y para muchos usuarios de Internet era una necesidad hacer contacto con otras personas allí en el telecentro. Las relaciones entre los contextos digital, arquitectónico y personal identificados van más allá de los límites de este artículo. Aquí nos detendremos para explorar las conversaciones digitales.

En los telecentros comunitarios, ¿qué hacen los internautas en su tiempo diario dedicado a Internet? En general, Piscitelli (2005) describe las características de uso de Internet en términos de escritura, lectura y el estudio en la red. En la Webgrafía realizada los telecentros, en términos de permanencia efectiva en el espacio digital, se registraron varios ambientes específicos o categorías ${ }^{6}$ en que navegaban 136 usuarios de Internet Arístides Vinholes, Telêmaco Borba (Faróis do Saber) y Vila Real (Paranavegar) (véase la Tabla 1).

${ }^{6}$ Registramos 3.064 minutos de navegación en Aristides Vinholes, correspondiente a 57 internautas (hasta $1 \mathrm{~h}$ por persona), en los días 03,11 e 26/10 y 01/11/2007; 1.856 minutos en Vila Real, referentes a 64 internautas (hasta 30 min por persona), en los días 04, 17, 18, 19 y 20/10/2007, y 760 minutos en Telêmaco Borba, correspondientes a 15 internautas (hasta $1 \mathrm{~h}$ por cada uno), en los días 13 y 14/10/2007.

Mediaciones Sociales, № 8, I semestre 2011, pp. 127-154. ISSN electrónico: 1989-0494. 
Tabla 1. Webgrafia - Ambientes o temáticas de navegación: tiempo de permanencia

\begin{tabular}{|c|c|c|c|c|c|}
\hline $\begin{array}{l}\text { Ambientes } \\
\text { específicos o } \\
\text { categorías }\end{array}$ & $\begin{array}{l}\text { Farol A. } \\
\text { Vinholes } \\
\text { 57 inter- } \\
\text { nautas }\end{array}$ & $\begin{array}{c}\text { Farol T. Borba } \\
15 \text { internautas }\end{array}$ & $\begin{array}{c}\text { Paranavegar } \\
\text { Vila Real } \\
64 \\
\text { internautas }\end{array}$ & $\begin{array}{l}\text { Total mi- } \\
\text { nutos }\end{array}$ & $\%$ \\
\hline Orkut & $967 \mathrm{~min}$ & $323 \mathrm{~min}$ & $1.119 \mathrm{~min}$ & 2.409 & 42,63 \\
\hline Meebo & $178 \mathrm{~min}$ & $72 \mathrm{~min}$ & $104 \mathrm{~min}$ & 354 & 6,26 \\
\hline $\begin{array}{l}\text { Chat instantá- } \\
\text { neo-Messenger }\end{array}$ & $183 \mathrm{~min}$ & $10 \mathrm{~min}$ & $103 \mathrm{~min}$ & 296 & 5,23 \\
\hline $\begin{array}{l}\text { Sitio de provee- } \\
\text { dores de e-mail } \\
\text { (msn, yahoo, } \\
\text { bol, uol) }\end{array}$ & $141 \mathrm{~min}$ & $35 \mathrm{~min}$ & $44 \mathrm{~min}$ & 220 & 3,89 \\
\hline $\begin{array}{l}\text { Sitios de depor- } \\
\text { te, juegos en } \\
\text { línea, entrete- } \\
\text { nimiento, TV }\end{array}$ & $971 \mathrm{~min}$ & $260 \mathrm{~min}$ & $322 \min$ & 1.553 & 27,48 \\
\hline $\begin{array}{l}\text { Sitio de búsque- } \\
\text { da (Google), } \\
\text { Pesquisas esco- } \\
\text { lares }\end{array}$ & $341 \mathrm{~min}$ & $30 \mathrm{~min}$ & $85 \mathrm{~min}$ & 456 & 8,07 \\
\hline $\begin{array}{l}\text { Editor de texto- } \\
\text { Open Office }\end{array}$ & $97 \mathrm{~min}$ & $\mathrm{O} \min$ & $\mathrm{O} \min$ & 97 & 1,71 \\
\hline $\begin{array}{l}\text { Sitio de Servicios } \\
\text { (CEF, Receta } \\
\text { Federal, Empleos, } \\
\text { Gobierno, Celular) }\end{array}$ & $151 \mathrm{~min}$ & $20 \mathrm{~min}$ & $66 \mathrm{~min}$ & 237 & 4,19 \\
\hline Blog & $7 \mathrm{~min}$ & $\mathrm{O} \min$ & $13 \mathrm{~min}$ & 20 & 0,35 \\
\hline Música & $28 \mathrm{~min}$ & $10 \mathrm{~min}$ & $\mathrm{O} \min$ & 38 & 0,67 \\
\hline Total & $3.064 \mathrm{~min}$ & $760 \mathrm{~min}$ & $1.856 \mathrm{~min}$ & 5.650 & $100 \%$ \\
\hline
\end{tabular}

Fuente: Lacerda (2008).

Mediaciones Sociales, № 8, I semestre 2011, pp. 127-154. ISSN electrónico: 1989-0494. DOI: $10.5209 /$ rev_MESO.2011.n8.6 
El tiempo de permanencia en Orkut en los tres telecentros llegó a 2409 minutos $(42,63 \%)$, seguido por los sitios con temas de deportes, juegos online, el entretenimiento y la TV con 1553 minutos (27,48\%). Los sitios de búsqueda y las investigaciones escolares llegaron a 456 minutos $(8,07 \%)$. Este aspecto y el relacionado con el uso de editor de textos $(97$ minutos $1,71 \%$ ) merecen algunas consideraciones sobre las particularidades del contexto del ambiente de los tres telecentros. La mayoría se queda en los motores de búsqueda y trabajos escolares o a utilizar el editor de textos en Aristides Vinholes (respectivamente, $341 \mathrm{~min}$ y $97 \mathrm{~min}$ ) en comparación con Telêmaco Borba (30 min / o min) y Vila Real ( 85 min / min o). Esto puede estar relacionado con el hecho del telecentro Aristides Vinholes estar situado en una plaza, rodeado de tres escuelas públicas, y la investigación se ha llevado a cabo durante la semana (de lunes a viernes).

Hicimos las encuestas en el telecentro de la Escuela Telêmaco Borba en el sábado y domingo, los dos días en que los Faróis do Saber ubicados en plazas, aislados de escuelas, se encuentran sin acceso a Internet. En estos dos días el foco del Farol Telêmaco Borba, ubicado en una escuela, es la comunidad en general. En el telecentro de Vila Real, no tenemos una escuela tan cerca y se han realizado estudios de campo tanto en días laborables como los sábados por la mañana. Hecha la consideración, es significativo señalar los ambientes que indicarían una mayor dedicación a las tareas escolares o la producción de programas de estudio, por ejemplo, si se combinan, no alcanzan el 10\% del tiempo dedicado al espacio digital en los tres telecentros investigados. Si tenemos en cuenta que Orkut, MSN, y Meebo ${ }^{7}$ son software sociales que ofrecen ambientes de socialización en red (Spyer, 2007), tenemos 3059 minutos (54,14\%) del tiempo de permanencia de los usuarios de Internet en el espacio digital de los tres telecentros destinados para lo que llamamos conversación digital.

Así pues, tenemos la conversación digital $(54,14 \%)$ y los sitios orientados al entretenimiento $(27,48 \%)$ representando un total de $81,62 \%$ del tiempo de permanencia de los usuarios de Internet en los telecentros Aristides Vinholes, Telêmaco Borba y Vila Real. La encuesta Ponline 2006 (Lidec-USP-Brasil) llevada a cabo en telecentros del Proyecto Acessa SP, del Gobierno del Estado de São Paulo, en Brasil, (Tabla 1B), y la investigación TIC Domicilios 2006 (Gráfico 1), del Comité Gestor de Internet

\footnotetext{
7 Plataforma desarrollada para Linux que engloba diversas herramientas conversacionales en un mismo suporte (MSN, Messenger do Yahoo!, etc.).
}

Mediaciones Sociales, № 8, I semestre 2011, pp. 127-154. ISSN electrónico: 1989-0494. DOI: 10.5209/rev_MESO.2011.n8.6 
de Brasil (CGI.Br), trajeron datos aproximados con mayor énfasis de los usuarios de Internet en el uso medios digitales conversacionales (correo electrónico, Orkut, MSN, chat, mensajeros, etc.).

Tabla 1B. Actividades realizadas en la Internet

\begin{tabular}{|l|c|}
\hline ¿Lo que haces en Internet? & Total \\
\hline Recibo y envío e-mail & $86,4 \%$ \\
\hline $\begin{array}{l}\text { Chatear vía mensajes instantáneos (ejemplo: MSN, Google Talk, } \\
\text { Gaim, ICQ) }\end{array}$ & $64,8 \%$ \\
\hline $\begin{array}{l}\text { Participo de sitios de comunidades de relacionamiento (ejemplo: } \\
\text { orkut) }\end{array}$ & $63,1 \%$ \\
\hline Leer noticias, periódicos y magazines & $47,9 \%$ \\
\hline Participo de salas de charlas/Chat & $45,4 \%$ \\
\hline Busco informaciones relacionadas a diversión e entretenimiento & $41,3 \%$ \\
\hline Busco trabajo o envío currículo & $36,6 \%$ \\
\hline Busco informaciones relacionadas a salud o a asistencia de salud & $25,1 \%$ \\
\hline Escucho radio y/o asisto videos/pantalla & $22,9 \%$ \\
\hline Juego videojuegos o juegos de computadora & $22,0 \%$ \\
\hline Hago download de juegos, músicas y softwares & $13,7 \%$ \\
\hline Hago compras o comparo precios & $12,1 \%$ \\
\hline Participo de listas o foros de discusión & $12,0 \%$ \\
\hline Acceso servicios bancarios & $6,6 \%$ \\
\hline Hago cursos a distancia & $4,5 \%$ \\
\hline Pago cuentas & $6 \%$ \\
\hline & \\
\hline
\end{tabular}

Fuente: Pesquisa Ponline 2006 - Acessa SP/Lidec/USP 


\section{Gráfico 1. Actividades realizadas en la Internet}

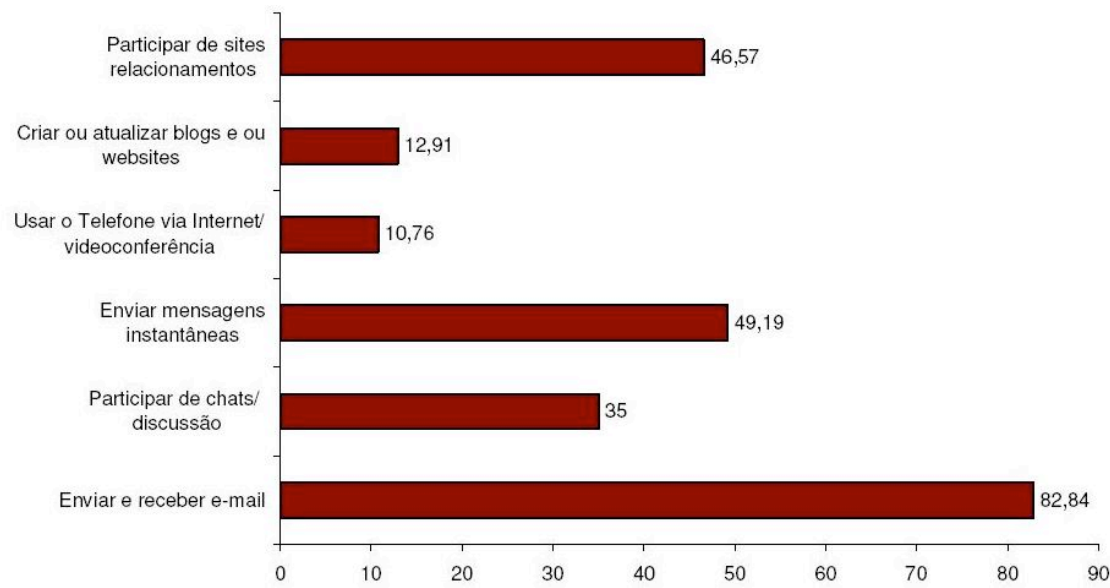

Base 2006: 2286 entrevistados que utilizaram a internet nos úlómos 3 meses para se comunicar. .).
Projeção populacional: 33 mïhōes de habitantes, com 10 anos ou mais de idade, segundo estimativa realizada com base na PNAD 2005.

Fuente: TIC Domicílios 2006 - Comitê Gestor da Internet do Brasil (CGIBR)

Cuando entramos en la materialidad de cómo los usuarios de Internet hacen uso de Orkut, por ejemplo, en los telecentros, encontramos una gran riqueza de significados mediados por las diferentes maneras en que viven y representan esta forma de conversación digital. Los mensajes de Orkut actúan como un sustituto para los mensajes $\mathrm{SMS}^{8}$, enviados para amigos o compañeros de trabajo con quién necesitan hablar, pero no encuentran todos los días. Gran parte de los jóvenes y adolescentes no tiene plata para comprar un teléfono móvil. Y cuando uno tiene un móvil, prefiere no exceder sus créditos de mensajes. Se produce una red solidaria de información sobre las actividades escolares y eventos. Y en la lectura de mensajes y la contemplación de las actualizaciones de álbumes de fotos personales encuentran una manera de sentirse más cerca de sus entes queridos que están lejos por alguna razón.

8 Short Message Service, servicio de mensajes cortos, de hasta 140 caracteres, enviadas entre teléfonos móviles.

Mediaciones Sociales, № 8, I semestre 2011, pp. 127-154. ISSN electrónico: 1989-0494.

DOI: 10.5209/rev_MESO.2011.n8.6 
“¡Para mí es todo! Es el único medio de conversación con gente que no se ve todos los días. (...) A veces hablamos de asuntos de escuela, una conversación variada" [Claudinha,9 14 años, internauta Paranavegar Vila Real, Curitiba, $14 / 11 / 2007]$.

"Debido a que usted tiene contacto con la gente, a sus amigos que no siempre se puede estar hablando en persona" [Johny, 17 años, internauta Paranavegar Vila Real, Curitiba, 07/11/2007].

"Cuando hace mucho tiempo de que yo no veo a la persona. Cuando no nos vemos en la escuela, algo así, le pregunto por qué no ha venido. Si tiene trabajos escolares, yo por lo menos aviso a mis colegas que va haber prueba a través de Orkut" [Wilton, 17 años, internauta Farol do Saber Aristides Vinholes, Curitiba, 08/11/2007].

"En mi Orkut, siempre estoy viendo a mis hermanos, siempre veo a mi madre. Me deja sentir próxima, porque yo no vivo con ellos. Me hace más cerca de ellos, así que ya no siento mucho la falta” [Angélica, 17 años, internauta Farol do Saber Aristides Vinholes, Curitiba, 08/11/2007].

Incluso los datos enviados por Celepar -empresa pública coordinadora del proyecto Paranavegar-sobre los tipos de uso elegidos por los usuarios (septiembre 2007), señalan, de un total de 159 acciones elegidas (programas), 102 de "ocio" (64,15\%), 55 para "otros" (34,59\%) y "investigación escolar” sólo dos (1,25\%). "Correo electrónico”, "búsqueda de empleo”, "Negocio" y "servicios públicos” no tuvieron ninguna previsión. ${ }^{10}$ Los datos para el mes de septiembre, del Departamento de Educación de la Ciudad de Curitiba, sobre las elecciones de tipo de uso por los usuarios de Internet en el Farol Aristides Vinholes también destacan el "ocio" con 860 programas de un total de 1.300 (66,15\%). Poco después, llega "búsqueda” con 336 (25,84\%), "escribir" con $71(5,46 \%)$ y "correo electrónico" con 32 (2,46\%). El proyecto Paranavegar tenía, en octubre de 2007, 43.819 personas registradas a través en toda la Provincia de Paraná (Brasil). Y todos los horarios programados para el período comprendido entre el 17/07/2006 al 03/10/2007 (Tabla 2), en todos los telecentros del proyecto, también hacen hincapié en el "ocio" (54,6\%), seguido por el uso del "co-

9 Todos los nombres son ficticios en las declaraciones con el fin de preservar la identidad de los participantes en la investigación.

10 Estos son los tipos de programación de servidor en la hoja de trabajo del telecentro, que genera los datos directamente a la Celepar. Telecentro de Curitiba - Vila Real - Informe Mensual Resumido - Período de 01/9/2007 hasta 30/9/2007. 
rreo electrónico" (29,5\%). La "investigación escolar" se limita a 5,29\% y la "búsqueda de trabajo", "servicios públicos" y los "negocios" ni siquiera representan el $1 \%$ de las reservas totales. El proyecto Faróis do Saber poseía, a finales de 2007, 26.126 usuarios registrados en la ciudad de Curitiba. El informe de octubre de 2007 (Tabla 3), producido por el Departamento de Educación de la Ciudad de Curitiba, señaló 23.293 (60,05\%) programaciones de horarios para la finalidad de "ocio", de un total de 38.788 . El uso del "correo electrónico" representó 4.966 (12,80\%), seguido de "búsqueda" con 4.073 (10,5\%) y "digitación” con 2.932 (7,55\%). El tema de "empleo/Curriculum Vitae" sólo llegó a 784 programas (2,02\%). Así que hay una cercanía entre los datos de permanencia en la espacialidad digital que hemos registrado, los datos locales (A. Vinholes y Vila Real) y los datos generales de programa en ambos proyectos de inclusión digital (Faróis do Saber y Paranavegar).

Tabla 2. Informe de los programas en telecentros Paranavegar

\begin{tabular}{|l|c|c|}
\hline Uso & Programas & $\%$ \\
\hline Correo electrónico & 133.853 & 29.5 \\
\hline Investigación Escolar: & 24.018 & 5.29 \\
\hline Búsqueda de Trabajo: & 3.058 & 0.67 \\
\hline Servicios Públicos: & 3.384 & 0.74 \\
\hline Ocio: & 24.7910 & 54.6 \\
\hline Negocios: & 1.845 & 0.40 \\
\hline Otros: & 39.285 & 8.66 \\
\hline Total de usos & 453.353 & 100 \\
\hline
\end{tabular}

Fuente: Celepar - Informe Consolidado de los Telecentros Paranavegar, 17/07/2006 hasta 03/10/2007. 
Tabla 3. Informe de los programas en telecentros del Proyecto Farol do Saber

\begin{tabular}{|l|c|c|}
\hline Uso & Programa & $\%$ \\
\hline Correo electrónico & 4.966 & 12,80 \\
\hline Búsqueda & 4.073 & 10,50 \\
\hline Digitar textos & 2.932 & 7,55 \\
\hline Ocio & 23.293 & 60,05 \\
\hline empleo/currículo & 784 & 2,02 \\
\hline imprimir & 849 & 2,18 \\
\hline scanner & 532 & 1,37 \\
\hline Jugar ajedrez & 416 & 1,07 \\
\hline entrenamiento & 561 & 1,44 \\
\hline becario & 247 & 0,63 \\
\hline Computadora con problema & 135 & 0,34 \\
\hline Total & 38.788 & 100 \\
\hline
\end{tabular}

Fuente: Departamento Municipal de Educación de Curitiba - Tipos de uso mensuales, Octubre de 2007.

Cabe señalar que los objetivos de los programas de inclusión digital ${ }^{11}$ como el gobierno electrónico, servicios bancarios, búsqueda de empleo, sólo alcanzaron a 237 minutos (4,19\%) en nuestra hoja de cálculo de la navegación (véase la tabla 2) y tuvieron resultados poco importantes, entre 0,5 y $2 \%$, en los datos del proyecto Paranavegar (Tabla 1) y Faróis do Saber (Tabla 2). Es probable que este bajo índice se relaciona con el grupo de edad específico del público (tabla 4) que hemos documentado su navegación en los tres telecentros. En el ambiente de los telecen-

\footnotetext{
${ }^{11}$ Defendidos por los países desarrollados y sus organismos multilaterales (ONU, UNESCO, la UNCTAD, el ICA IRCD-, Banco Mundial, OCDE, etc.).
} 
tros investigados, hay una presencia sobre todo de adolescentes y niños. Y esto no es una particularidad de los espacios investigados.12 De los 136 usuarios de Internet que han tenido su navegación asignada, 29,41\% eran niños, 54,41\% adolescentes, $19,85 \%$ eran jóvenes y sólo $8,08 \%$ eran adultos. Por lo tanto, el grupo de los que usan servicios públicos en línea o que están en busca de trabajo, por ejemplo, correspondió a 27,93\% de usuarios de Internet que tuvieron su navegación en el espacio digital asignada en la investigación de campo. Con respecto al género (Tabla 5), tuvimos un poco de equilibrio, con 44,85\% mujeres y 55,15\% hombres.

Tabla 4. Grupo de edad de los internautas asignados en la navegación

\begin{tabular}{|l|c|c|c|c|c|}
\hline & A. Vinholes & T. Borba & Vila Real & total & \% \\
\hline Niños $^{13}$ & 21 & 4 & 15 & 40 & 29,41 \\
\hline Adolescente & 16 & 7 & 35 & 74 & 54,41 \\
\hline Joven & 15 & 3 & 9 & 27 & 19,85 \\
\hline Adulto & 5 & 1 & 5 & 11 & 8,08 \\
\hline Total & 57 & 15 & 64 & 136 & 100 \\
\hline
\end{tabular}

Fuente: Lacerda (2008).

Tabla 5. Género de los internautas asignados en navegación

\begin{tabular}{|l|c|c|c|c|c|}
\hline & A. Vinholes & T. Borba & Vila Real & Total & $\%$ \\
\hline Femenino & 23 & 8 & 30 & 61 & 44,85 \\
\hline Masculino & 34 & 7 & 34 & 75 & 55,15 \\
\hline Total & 57 & 15 & 64 & 136 & 100 \\
\hline
\end{tabular}

Fuente: Lacerda (2008).

${ }^{12}$ En la investigación Ponline 2006 (Lidec-USP), los participantes de los telecentros Acessa SP encuestados $52 \%$ eran adolescentes y preadolescentes.

${ }^{13}$ Nosotros no trabajamos con grupos de edad en la asignación. La clasificación fue impresionista, basada en el tamaño físico y la conducta expresada por los usuarios de Internet que buscan en el registro.

Mediaciones Sociales, № 8, I semestre 2011, pp. 127-154. ISSN electrónico: 1989-0494.

DOI: 10.5209/rev_MESO.2011.n8.6 
Los datos del mapa de navegación de los 136 usuarios de Internet tienen muchas similitudes a los datos obtenidos para los tres sitios más visitados (Tabla 6) por 81 usuarios de Internet que respondieron al cuestionario en los telecentros A. Vinholes, Telêmaco Borba y Vila Real. El Orkut junto con Meebo fueron nombrados 69 veces $(85,18 \%)$, seguido de MSN/Hotmail con 34 indicaciones (41,95\%) y del Google con 28 $(34,56 \%)$, a la vez que juegos, música y humor quedaron muy cercanos con 27 direcciones $(33,33 \%)$. Los proveedores de correo electrónico se les dio 20 veces $(24,69 \%)$. Los servicios (bancos, puestos de trabajo, el gobierno, telefonía) fueron algo mayores ( 11 veces, $13,58 \%$ ) a los periódicos, radio y televisión que recibieron nueve nominaciones (11,11\%). Es interesante que Google fuera separado de los demás motores de búsqueda, que sólo obtuvieron cuatro asignaciones $(4,93 \%)$.

Tabla 6. Hable los tres sitios que usted más suscribe:

\begin{tabular}{|l|r|r|}
\hline Sites por categoría & \multicolumn{1}{|c|}{ Total } & \multicolumn{1}{c|}{$\%$} \\
\hline Orkut/Meebo & 69 & 85,18 \\
\hline Google & 28 & 34,56 \\
\hline MSN - Hotmail & 34 & 41,97 \\
\hline You Tube & 9 & 11,11 \\
\hline Juegos, Música, Humor & 4 & 33,33 \\
\hline Sitios de búsqueda & 20 & 24,69 \\
\hline Sitios de proveedor de correo electrónico & 11 & 13,58 \\
\hline $\begin{array}{l}\text { Servicios(Banco, Gobierno, puestos de } \\
\text { trabajo, Telefonía) }\end{array}$ & 9 & 11,11 \\
\hline Periódicos, TV y Radio & & \\
\hline
\end{tabular}

Fuente: Lacerda (2008).

De los 81 internautas, 40 eran del sexo femenino y 40 del masculino, uno no ha señalado el género. En el grupo de edad (Tabla 7), hay un pre- 
dominio de internautas de 10 hasta 15 años (33) y de 15 hasta 20 años de edad (29).

Tabla 7. Grupo de edad de la encuesta

\begin{tabular}{|l|c|c|}
\hline Grupo de Edad & Total & \% \\
\hline 10 a 15 años & 33 & 40,74 \\
\hline 15 a 20 años & 29 & 35,80 \\
\hline 20 a 25 años & 11 & 13,58 \\
\hline 25 a 30 años & 1 & 1,23 \\
\hline 30 a 40 años & 6 & 7,40 \\
\hline
\end{tabular}

Fuente: Lacerda (2008).

Los trayectos desarrollados por internautas, como hábitos de navegación en la Internet, son en realidad bastante limitados en comparativo a lo que pueden tener acceso en el espacio digital a través del ambiente del telecentro, pero siempre hay hendeduras para otras rutas. Los medios de comunicación conversacionales -señalados tanto en datos cuantitativos cuanto en la observación cualitativa presencial- también son punto de partida para la navegación. Lo primero que hace la entrevistada Claudinha, cada vez que va al telecentro, es visitar Orkut y, a continuación, abre el MSN. Entonces entran en escena sitios de busca de puestos de trabajo. "Estoy en busca de un trabajo temporal para ayudarla [a su madre]. Así que esas son las tres ventanas que abro todos los días que vengo aquí: la de Orkut, de MSN y de las pasantías"14. El entrevistado Johny siempre entra primero en Orkut y, a continuación, en MSN. La entrevistada Angélica suele mantener abiertos MSN y Orkut mientras está en línea, ya que mejora sus posibilidades de encontrar a sus amigos: "Yo estoy siempre con ambos abiertos. Así que si mi amigo está fuera de línea en MSN, entonces voy a hablar con él a través de Orkut"15. Como el tiempo del entrevistado Emanuel es reducido y necesita dedicar una parte de su período a las acti-

${ }^{14}$ Entrevista con Claudinha, 14 años, internauta Paranavegar Vila Real, Curitiba, 14/11/2007.

${ }^{15}$ Entrevista con Angélica, 17 años, internauta Farol do Saber Aristides Vinholes, Curitiba, 08/11/2007.

Mediaciones Sociales, № 8, I semestre 2011, pp. 127-154. ISSN electrónico: 1989-0494. DOI: $10.5209 /$ rev_MESO.2011.n8.6 
vidades del curso técnico, su turno en el telecentro Aristides Vinholes es dedicado principalmente a ver su correo electrónico y tener contacto con otras personas. Los ambientes digitales a los que él tiene más acceso son Google, Yahoo!, además de los sitios de dos importantes diarios brasileños: Folha de São Paulo y O Estado de São Paulo.

"Puedo usar sitios como Google, Yahoo, Folha de S. Paulo, Estado de S. Paulo... Los uso, sobre todo, como fuente de investigación. Uso Google, según el trabajo que tenga que hacer, escribo allí y veo sitios relacionados. Pero para lo que más lo uso es para ver el correo electrónico... Tener contacto con otras personas. Eso es lo que más uso allí...” [Emanuel, 24 años, internauta Farol do Saber Aristides Vinholes, Curitiba, 01, 08 y 09/11/2007].

En sus estrategias para la navegación, los encuestados admitieron navegar sin rumbo en varias ocasiones por el espacio digital. Esto es lo que Lévy (1999) llama de saqueo y Lemos (2001) nombra de flanerie. Los espectadores ven esta práctica como una oportunidad para descubrir nuevos sitios, nuevas cosas. Fue navegando sin un destino cómo el entrevistado Wilton encontró el sitio del Jornal de Poesia ${ }^{16}$ con textos de varios poetas nacionales (brasileños) y comenzó a frecuentarlo, o sea, convirtió el sitio en parte de su mapa de navegación. Pero cuando construyen un cierto hábito, reducen o detienen la navegación al azar. La entrevistada Angélica, cuando no tenía MSN u Orkut, navegaba sin un destino, pues no tenía nada que hacer. Hoy en día, va directa al grano: MSN y Orkut ${ }^{17}$. La entrevistada Claudinha, cuando se satura de mirar el Orkut, pasa a navegar por la internet sin una meta definida. Ella va a Google, pero tiene su propia estrategia para flanear (dar un paseo): intenta recordar algún tema interesante de la lección de clase o algo que ha oído comentar a alguien y empieza a buscarlo cuando le viene a la mente. El entrevistado Emanuel ejercita mucho esta práctica porque, a menudo, se olvida de lo que vino a hacer o encuentra algo que acaba por llamar su atención, aunque no siempre se considera importante. Admite que a veces corre el riesgo de rebasar el período permitido de una hora de navegación y que cuando, por fin, encuentra algo relevante se le ha acabado el tiempo. Así, el ambiente escolar, las temporalidades y las relaciones interpersonales son mediaciones importantes presentes en las estrategias de navegación.

${ }^{16}$ Disponible en http://www.secrel.com.br/jpoesia/poesia.html

${ }^{17}$ Entrevista con Angélica, 17 años, internauta Farol do Saber Aristides Vinholes, Curitiba, 08/11/2007.

Mediaciones Sociales, № 8, I semestre 2011, pp. 127-154. ISSN electrónico: 1989-0494. 
"Haciendo click en un enlace relacionado, encontré un sitio llamado, si no me equivoco, Jornal de Poesia. Y he visto que en este sitio tiene varios poemas de diversos autores procedentes de Brasil. Muchos autores y muchos buenos poemas. Me gustó, es interesante" [Wilton, 17 años, internauta Farol do Saber Aristides Vinholes, Curitiba, 08/11/2007].

"Ah, a mí me sirve para descubrir cosas nuevas. Por ejemplo, en unos días preguntaré a los chicos cómo descargar juegos, porque el juego original cuesta 200 reales. Por lo tanto, estoy viendo cómo obtener ese juego. Así que estoy buscando, me entero de varias cosas" [Johny, 17 años, internauta Paranavegar Vila Real, Curitiba, 07/11/2007].

"Por lo general, cuando me enfermo de Orkut, lo hago. Entonces copio y pego en la búsqueda de Google y pesquiso cualquier idea que venga en mi cabeza. ‘Maldita sea! necesito saber acerca de’..., 'mi profesor dijo tal cosa hoy', escribo allí. Háganos saber acerca de lo que es... Creo que es.... A continuación, basta hacer un clic y usted va a pensar cosas diferentes, es legal" [Claudinha, 14 años, internauta Paranavegar Vila Real, Curitiba, 14/11/2007].

\section{COMPleJo ECOSISTEMA COMUNICACIONAL}

Una observación sólo cuantitativa pone de relieve la gran popularidad de los medios de conversación. Pero sólo la mirada cuantitativa no basta para comprender la complejidad con que los usuarios de Internet atribuyen sentidos a sus prácticas: características de usos, valoraciones, jerarquías, roles, intenciones y sentimientos de cada uno. Y todo esto produce un complejo ecosistema de comunicación mediada por la semiosfera personal de cada usuario. Estas formas de articulación y atribución de sentido constituyen una racionalidad, como tecnicidad. Se desarrollan como sabidurías producidas en la experiencia digital de la vida cotidiana (Pérez Tapias, 2003). Para la entrevistada Claudinha, el Orkut es para hablar con la gente sin la necesidad de que la respuesta sea inmediata. Ya el MSN es para los problemas que deben resolverse ahora. Ambos son para hablar con personas que no tienen contacto presencial, pero no muy a menudo. El correo electrónico es más importante para las interacciones que deben ser almacenadas. "Y cuando el tema es serio, es el correo electrónico". Es su memoria digital. Viene a sustituir a la interacción por teléfono fijo (el teléfono fijo al que tienen acceso es de sus tíos). "Informo a la gente de mi correo electrónico cuando me preguntan el número de teléfono, por lo general, les paso mi email. El correo electrónico es para comunicaciones

Mediaciones Sociales, № 8, I semestre 2011, pp. 127-154. ISSN electrónico: 1989-0494. DOI: 10.5209/rev_MESO.2011.n8.6 
mayores o cosas más importantes, se quedan almacenadas.” Para cada tipo de persona, Johny tiene una forma específica de producir sus mensajes en Orkut.

"Depende de la persona. Cuando, por ejemplo, voy a hablar con mi tía, voy allí y digo más cosas de la familia. Y cuando hablamos entre amigos tenemos una manera propia de entendernos. Por lo tanto, si hablo con una persona desconocida, voy más despacio. Soy más educado para ver cómo la persona va a reaccionar" $[$ Johny, 17 años, internauta Paranavegar Vila Real, Curitiba, $07 / 11 / 2007]$.

En el telecentro se usa el sistema Linux y sólo es posible hacer uso del MSN a través de la plataforma Meebo. Pero la plataforma Meebo no permite intercambiar archivos. El entrevistado Johny lamenta no poder hacer intercambio de archivos por la incompatibilidad entre los sistemas Linux y Windows. "Una persona, ayer, me envió una foto. Pero el sistema me ha negado el acceso, pues la persona usa el sistema Windows y yo tengo el Linux". Johny utiliza el correo electrónico Yahoo! para interactuar con otras personas porque puede almacenar en diferentes carpetas y es uno de los más populares, de fácil reconocimiento cuando se va a pasar la dirección para uno. También tiene un correo electrónico en Gmail (Google), pero que lo utiliza para los materiales de archivo, tiene suficiente memoria. "El de Yahoo! se conoce las letras, si hablas con la gente sobre Yahoo! la persona ya sabe cómo escribir, ¿entiendes? En Gmail la persona tiene dudas, es decir, de escribir. Por lo tanto, yo también uso mucho Gmail, pero para el almacenamiento mismo”. El correo electrónico termina reemplazando el papel del teléfono para Johnny, en la tentativa de encontrar un trabajo. "Yo llamé. Ella pasó su correo electrónico. Por lo tanto, no puedo llamar a todo momento, porque no tengo condiciones. Por lo tanto, yo vengo, voy en Internet y envío e-mail a ella”.

El entrevistado Wilton utiliza Orkut específicamente para enviar mensajes a sus amigos, incluso aquellos que sólo conoce de forma digital. Por lo tanto, ve la importancia de las imágenes en el álbum de Orkut: "Tal vez para que las personas puedan conocerme mejor a mí, ¿entiendes? Hay gente que sólo conozco a través de Orkut"18. Él clasifica el tipo de situación en que produce los mensajes para Orkut: "Cuando hace tiempo que yo no veo a la persona, cuando no nos vemos en la escuela, me pregunto ¿por

${ }^{18}$ Entrevista con Wilton, 17 años, internauta Farol do Saber Aristides Vinholes, Curitiba, 08/11/2007.

Mediaciones Sociales, № 8, I semestre 2011, pp. 127-154. ISSN electrónico: 1989-0494. DOI: 10.5209/rev_MESO.2011.n8.6 
qué no ha venido? Si tiene trabajo escolar, yo por lo menos informo a mis colegas que hay examen y tarea escolar". El MSN es para él un hobby. Wilton tiene dos direcciones de e-mail debido a problemas para acceder a Gmail en el Farol Aristides Vinholes, dijo. En el telecentro usa Yahoo! "La función principal de mi correo electrónico es enviar tareas de clase para mis colegas". Recoge el material en el telecentro y se lo envía. O también enviar a sí mismo los resultados de la búsqueda en el telecentro para abrirlo en su casa en el fin de semana. Utiliza Internet por línea telefónica en casa.

La entrevistada Angélica utiliza MSN para encontrar amigos que estén conectados y chatear con su padre, que vive en el Nordeste de Brasil. El Orkut le hace sentirse cerca de su madre y hermanos, al ver las fotos en su perfil. El correo electrónico es para intercambio de tareas escolares con colegas y amigos y para recibir informaciones más oficiales, como una confirmación de su registro de vestibular, que se llevó a cabo sólo por correo electrónico. "Mi amiga encuentra un texto y me lo envía a mi correo electrónico. Voy allí y miro si está bien. Sí sí, nos juntamos para hacer el trabajo. [...] Así que es importante que usted tenga un e-mail porque hace su vida más fácil. Usted hace muchas cosas a través de él”19.

Emanuel utiliza Orkut como una manera de encontrar a antiguos colegas de clase: "Aquí descubro a muchos colegas míos de la escuela, hay tiempos que no los podía ver" 20 . Pero él dijo que no utiliza el Orkut en el telecentro, sólo cuando va a una lan house. Es crítico con la utilización del Orkut en telecentros. Por lo tanto, Emanuel no usa allí el Orkut para mantener la coherencia. Usa MSN para hablar con su hermano, que vive en São Paulo, ya que el dispositivo permite saber quién está conectado. Sólo que tampoco utiliza esta forma de conversación digital en el telecentro, porque no se puede usar audio y vídeo en la interacción. "Facilítame a verlo a través de la pantalla. Dame contacto con él allí directamente. Si tengo un ordenador encendido, él sabe que yo estoy ahî". Él usa correo electrónico para recibir apostilla del curso de técnicas de seguridad. También recibe, por correo, catálogos de nuevos productos que serán ofrecidos en el almacén de materiales de construcción en que trabaja. "Tengo que ser

${ }^{19}$ Entrevista con Angélica, 17 años, internauta Farol do Saber Aristides Vinholes, Curitiba, 08/11/2007.

${ }^{20}$ Entrevista con Emanuel, 24 años, internauta Farol do Saber Aristides Vinholes, Curitiba, 01, 08 y $09 / 11 / 2007$. 
consciente de lo que vendo. Él envía por e-mail y tengo que abrir el correo electrónico, a través del editor de texto”.

\section{Consideraciones Finales}

Así pues, tenemos la conversación digital (54,14\%) y sitios orientados al entretenimiento $(27,48 \%)$ sumando $81,62 \%$ de tiempo de la estancia de los internautas observados en los telecentros. Los resultados son aproximados de los de las encuestas del proyecto Acessa SP (Gobierno de São Paulo) y de la TIC Domicílios 2006, del Comité Gestor de Internet Brasileño (CGI.Br), que señalaron gran énfasis en el uso de medios digitales conversacionales (correo electrónico, Orkut, MSN, chat, mensajeros, etc.) por los usuarios de Internet en telecentros. Para los niños, adolescentes y jóvenes, el tiempo de conversación digital no se entiende en su racionalidad técnica como "tiempo perdido" o de "ocio sin preocupaciones". Los medios de comunicación conversacionales son un sustituto de las tecnologías más caras para la interacción, como los mensajes SMS. Producen una red solidaria de información sobre las actividades escolares o de grupo. Fortalecen las redes de sociabilidad locales y fortalecen los lazos con parientes lejanos o amigos. Y es evidente que muchos de los objetivos defendidos por los países desarrollados y sus organismos multilaterales (ONU, UNESCO, la UNCTAD, el ICA IRCD, el Banco Mundial, OCDE) como el gobierno electrónico, servicios financieros, búsqueda de empleo, etc., no dialogan con las mediaciones constitutivas de la tecnicidad digital de los usuarios de Internet en la localidad. Las políticas están aún lejos de los jóvenes y los adolescentes, para quienes, en la mayoría de los planes, los telecentros no son estratégicamente pensados. Salvo en el caso del Telecentro Vila Real, en que la propia comunidad hizo crear el telecentro para ofrecer un espacio de interacción y socialización para los niños, adolescentes y jóvenes. Pero esto no es una política efectiva de la Red Paranavegar, del Gobierno del Estado de Paraná (Brasil). Identificamos que en el ambiente de los telecentros investigados es predominante la presencia de los niños y adolescentes. Este fenómeno se repite en los proyectos de inclusión digital ${ }^{21}$. Así, tenemos dos tipos distintos de tecnicidad como mediación. Por primero, la racionalidad técnica de los responsables por los programas de inclusión digital, cuyo lugar de significación es de la competen-

${ }^{21}$ En la pesquisa Ponline 2006 (Lidec-USP), de los participantes de telecentros del proyecto Acessa $S P$ que contestaron la encuesta $52 \%$ eran adolescentes e preadolescentes.

Mediaciones Sociales, № 8, I semestre 2011, pp. 127-154. ISSN electrónico: 1989-0494. DOI: 10.5209/rev_MESO.2011.n8.6 
cia, de la efectividad del uso con fines de prácticas instrumentales de cualificación laboral o aplicaciones educativas tradicionales de aprendizaje. Por otra parte, tenemos la racionalidad técnica de los niños y adolescentes en sus modos de apropiación de los telecentros, como lugar de juego de interacciones sociales, de conversación, de ponerse en contacto, de pasatiempo colectivo o individual, de solidaridades heterogéneas. Por lo tanto, es necesario repensar las políticas de inclusión digital en Brasil con el fin de satisfacer las aspiraciones de la tecnicidad propia de estos sujetos representativos.

\section{Bibliografía}

AsSUMPÇão, Rodrigo O. D. (2001): Além da inclusão digital: o projeto sam-pa.org. Dissertação (Mestrado em Ciências da Comunicação). São Paulo: Universidade de São Paulo.

Bateson, Gregory (1998): Pasos hacia uma ecologia de la mente. Buenos Aires: Lohlé Lúmen.

Bourdieu, Pierre (2003): “A economia das trocas simbólicas", en Bourdieu, Pierre: As razões práticas. São Paulo: Papirus, pp. 157-197.

CASTELLS, Manuel (2003): La Galáxia Internet: reflexiones sobre Internet, empresa y sociedad. Barcelona: Debolsillo.

CASTELLS, Manuel (2004): "A Internet e a sociedade em rede", en OliveIra, José Paquete de; CARDOSo, Gustavo L.; BARREIRos, José Jorge: Comunicação, Cultura e Tecnologias de Informação. Lisboa: Quimera, pp. 22 1-244.

Certeau, Michel (2000): A invenção do cotidiano: Artes de fazer. Petrópolis: Vozes ( $5^{\mathrm{a}}$ ed.).

GiTLIN, Todd (2005): "Estilos de navegación e implicaciones políticas", en GiTuIN, Todd: Enfermos de información. Barcelona: Paidós, pp. 145-212.

HeidegGer, Martin. (2001): "La pregunta por la técnica”, en HeidegGer, Martin: Conferencias y artículos. Barcelona: Editores del Serbal, pp. 9-32.

JOHnson, Steve (2001): Cultura da interface. Rio de Janeiro: Zahar.

\footnotetext{
Mediaciones Sociales, № 8, I semestre 2011, pp. 127-154. ISSN electrónico: 1989-0494. DOI: 10.5209/rev_MESO.2011.n8.6
} 
LACERDA, Juciano de Sousa (2008): Ambiências comunicacionais e vivências midiáticas digitais. Tese de Doutorado. São Leopoldo: UNISINOS.

LOADER, B. (2004): "Apoio comunitário virtual? A política Social e a emergência do apoio social mediado por computador", en OliveIRA, José Paquete de; Cardoso, Gustavo L.; Barreiros, José Jorge: Comunicação, Cultura e Tecnologias de Informação. Lisboa: Quimera, pp. 127-160.

Lotman, Iuri M. (1996): “Acerca de la semiosfera”, en Lotman, Iuri M.: La semiosfera I. Madrid: Cátedra, pp. 21-42.

Martín-BARBero, Jesús (2001a): Dos meios às mediações: comunicação, cultura e hegemonía. Rio de Janeiro: UFRJ ( $2^{\mathrm{a}}$ ed.).

Martín-BARbero, Jesús; Rey, Gérman (2001b): Os exercícios do ver. São Paulo: Senac.

MARTÍN-BARBERo, Jesús (2004): "Razón técnica y razón política: espacios/tiempos no pensados", Revista Latinoamericana de Ciencias de la Comunicación, año $1, \mathrm{n}^{\circ}$ 1, julio-diciembre de 2004, pp. 22-37.

Menou, Michel J.; Delgadillo Poepsel, Karin; Stoll, Klaus (2004): "Latin American Community Telecenters: 'It's a long way to TICperary", The Journal of Community Informatics, vol. 1, ed. 1, pp. 39-57. Disponible en: http://www.cijournal.net/index.php/ciej/article/view/191/146. Consultado el 10 de noviembre de 2007.

Oliva, Rafael (2003): “Acessa São Paulo: promovendo a inclusão digital”, Instituto para a Conectividade nas Américas (ICA). São Paulo, Março, 2003. Disponible en: http://www.idrc.ca/es/ev-106951-201-1-DO_TOPIC.html. Consultado el 17 de octubre de 2005.

Orozco GómEz, Guillermo (2006). "Comunicação social e mudança tecnológica: um cenário de múltiplos desordenamentos”, en MoraEs, Denis (org.): Sociedade midiatizada. Rio de Janeiro: Mauad, pp. 81-98.

PÉREZ TAPIAS, José Antonio (2003): Internautas y náufragos: la búsqueda del sentido en la cultura digital. Madrid: Trotta.

PISCITELLI, Alejandro (2005): Internet, la imprenta del siglo XXI. Barcelona: Gedisa.

Robinson, Scott. S (2000). "Telecentro en México: desaios y posibilidade”, en FinQUELEVICH, S. (org.): Ciudadanos, a la Red! Los vínculos sociales en el ciberespacio. Buenos Aires: Ciccus-La Crujia, pp. 117-136.

Mediaciones Sociales, № 8, I semestre 2011, pp. 127-154. ISSN electrónico: 1989-0494. DOI: 10.5209/rev_MESO.2011.n8.6 
Santos, Milton. (2002): A natureza do espaço. São Paulo: Edusp.

SILverRA, Sérgio Amadeu (2005): Exclusão digital: a miséria na era da informação. São Paulo: Perseu Abramo.

Silverstone, Roger (2002): Por que estudar a mídia?. São Paulo: Loyola.

SPYER, Juliano (2007): Conectado: o que a internet fez com você e o que você pode fazer com ela. Rio de Janeiro: Zahar.

WARSChaUer, Mark (2006): Tecnologia e inclusão social: a exclusão digital em debate. São Paulo: Senac. 


\section{PARA CITAR ESTE TRABAJO EN BIBLIOGRAFÍAS:}

LACERDA, Juciano de Sousa (2011): "Conversaciones digitales: las mediaciones del uso de la Internet en los telecentros de los proyectos Faróis do Saber y Paranavegar", Mediaciones Sociales. Revista de Ciencias Sociales y de la Comunicación, $\mathrm{n}^{\mathrm{o}}$ 8, pp. 127-154. DOI: 10.5209/rev_MESO.2011.n8.6; http://dx.doi.org/10.5209/rev MESO.2011.n8.6

\section{${ }^{(*)}$ El autor}

Juciano de Sousa Lacerda es periodista y profesor del Departamento de Comunicación Social de la Universidade Federal do Rio Grande do Norte. Es parte del cuadro de profesores permanentes del Programa de Postgrado Estudos da Mídia, en la línea de investigación "Estudio de los media y las prácticas sociales". Doctor en Ciencias de la Comunicación de la Universidade do Vale do Rio dos Sinos (2008). Tiene pasantía de doctorado en la Universidad Autónoma de Barcelona (10/2005 a 03/2006). Es vicecoordinador del Grupo de Investigación de la Comunicación para la Ciudadanía de la Sociedad Brasileña de Estudios Interdisciplinarios de la Comunicación (INTERCOM). Es jefe adjunto del Grupo de Investigación Pragmática de la Comunicación y de los Media (Pragma-UFRN) y participa de la Red AMLAT - Red Temática de Cooperación Científica en Comunicación, Ciudadanía, Educación e Integración en Latinoamérica - Prosul. MCT / $\mathrm{CNPq} \mathrm{N}^{\circ} 11 / 2008$, que involucra a grupos de investigación de Brasil, Argentina, Ecuador y Venezuela. Integra acciones de investigación en red con los Grupos de Investigación Procesos: Procesos Comunicacionales (CNPq/UNISINOS/Brasil) y del GrupCiber (Grupo de Investigación en Ciberantropologia) del Laboratorio en Antropología Social de la UFSC (Brasil).

RECIBIDO: 17 de septiembre de 2010. ACEPTADO: 29 de noviembre de 2010. 